\title{
An accurate signature verification system based on proposed HSC approach and ANN architecture
}

\author{
Mustafa S. Kadhm ${ }^{1}$, Mamoun Jassim Mohammed ${ }^{2}$, Hayder Ayad ${ }^{3}$ \\ ${ }^{1}$ Computer Engineering Techniques, Imam Ja'afar Al-Sadiq University, Baghdad, Iraq \\ ${ }^{2}$ Department of Computer Engineering, College of Engineering, Al-Iraqia University, Baghdad, Iraq \\ ${ }^{3}$ Department College of Business Administration, AL-Bayan University, Baghdad, Iraq
}

\section{Article Info}

Article history:

Received May 7, 2020

Revised Jun 9, 2020

Accepted Jul 25, 2020

\section{Keywords:}

ANN

HSC

Image thinning

ReLU

Signature verification

\begin{abstract}
With the rapid development of technology in all life fields, and due to the huge daily needs for banking systems process, documents processing and other similar systems. The authentication became more required key for these systems. One of the successful system to verify the any person is the signature verification system. However, a reliable and accurate system is still needed. For this reason, the security challenge is take place via authentic signatures. However, a reliable and accurate system is still needed. For this reason, the security challenge is take place via authentic signatures. Therefore, this paper present a reliable signature verification system using proposed histogram of sparse codes (HSC) feature extraction approach and artificial neural networks (ANN) architecture for classification. The system achieved fast computing $0.09 \mathrm{~ms}$ and accurate verification results that is 99.7\% using three different signature images datasets CEDAR, UTSig, and ICDAR.
\end{abstract}

This is an open access article under the CC BY-SA license.

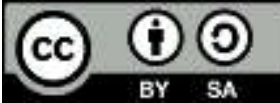

Corresponding Author:

Mustafa Salam Kadhm

Department of Computer Engineering Techniques

Imam Ja'afar Al-Sadiq University, Qahira, Baghdad, Iraq

Email: muit.salam@ sadiq.edu.iq

\section{INTRODUCTION}

Biometric system is the most effective system in many fields. Security of many systems are depends on the biometrics via several methods, such as finger, iris, signature, and etc [1]. It helps to identify person through its unique features and avoid unauthentic access to the system. Signature is the one of most powerful method for verification of the person. The signature verification system is widely used for identify the people via their handwritten signatures [2]. Many transactions for money around the world are occur daily. A powerful authenticated operations for this transactions are needed. Besides that, the signature could be faked by other people that try to claim the real signature personality and signatures [3].

The signature verification system detect the genuine and forgery signatures to provide an authenticated banking process. Two types of signature verification system are exist which are offline and online system. In recent years, offline systems are applied in many fields such as the cloud, and was appropriate than other biometrics methods $[4,5]$. We will focus on the offline system that use the scanned documents, bank cheques and vouchers in many applications. The offline type is more challenged because the online has a complete information and features that make forgery signatures difficult [6]. However, the offline has the signature images issue with noise, poor resolutions and other image processing issue. Several researches using different methods are done for signature verifications [7-9], but it still an active research file due to the increasing in the security challenges [10]. In this paper an efficient image preprocessing is 
performed to prepare the signature image for the feature extraction. HSC is employed in the best manner to extract the only desired features that represent the signature based on the sparse codes. Las, a proposed ANN arthiticture then applied for better verification accuracy. The rest of the paper organize as following: Section 2 describes the recent state of art systems for the signature verification, Section 3 the proposed system is explained in details. The experiments and evaluation are given in Section 4. Section 5 stated the conclusion of this paper.

\section{RELATED WORKS}

In below the most recent researches related to the signature verification system are viewed and briefly discussed. Anamika J., et al. [11], present a simple classification architecture for handwritten signature verification system called hallow convolutional neural network $(\mathrm{sCNN})$. The sCNN learn the signature features automatically. The authors also, provide two signatures datasets called CVBLSig-V1 and CVBLSig-V2 within 137 and 467 people respectively. The authors mentioned that, their system achieved a high accuracy and low matching error for their datasets and other public signature datasets. Jivesh P., et al., [12], proposed signature recognition and corner detection system using several methods. They used Surf and Harris algorithms for extracting the features. Besides, $\mathrm{CNN}$ is used for the classification the signatures. The system achieved $90-94 \%$ accuracy for signature recognition.

Tomasz H., et al., [13], proposed a long term verification system for the signature using block-chain schema and shell model. The system allow the maintaining for the signature validation without using the timestamps. The authors mentioned that, their system works well within large number of signed documents. Amruta B. et al., [14], represent siamese neural network (SNN) using CNN as subnetwork for signature verification. The network architecture shares same weights among number of sub networks inside it. The network generate an embedding vector with statistical metrics for robustness. Their system obtained a high accuracy in terms of FAR and FRR comparing to the state of art works.Pradeep N., [15], apply support vector machine (SVM) and shape correspondence methods for offline signature verification. The authors employ adaptive weighted combination of Euclidean distance and shape context distance for correspondence the pixels. After that, plate spline transformation is used for query signature plane transformation to reference signature plane. The system achieved an accuracy of $89.58 \%$ using SVM classifier and GPDS signature dataset. Victoria R., et al., [16], proposed an effective solution for random forgeries in a writer-independent context problem of signature verification using SNN. The SNN is trained with combinations of synthetic data and GAVAB dataset in order to produce infinite number of synthetic signatures. A high verification results are obtained using various signature datasets. Most of the existing systems focused on the classification stage of the signatures. Authors took the advantages of using the DNN and its powerful results. However, preprocessing and features extraction stages are ignored by most of the researchers. Furthermore, according to the descussed systems related to signature verification, we can observe that the accuracy and reliability are the mian challenges. This, makes the signature verification still an open research issue.

\section{PROPOSED SYSTEM}

Several processes are performed in the proposed work in order to get a better verification results. These processes are: 1) Image preprocessing, 2) feature extraction and 3) classification and verification. A complete view of the proposed work is illustrated in Figure 1.

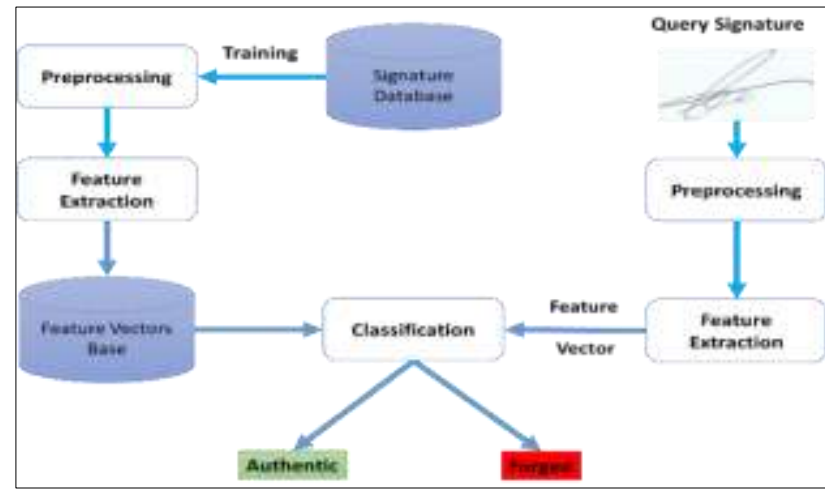

Figure 1. Proposed signature verification system

Indonesian J Elec Eng \& Comp Sci, Vol. 21, No. 1, January 2021 : 215 - 223 


\subsection{Preprocessing}

In preprocessing, a thinned [17] signature image is produced. This image then passed for the feature extraction process. The purpose of this idea is to reduce the image dimension and eliminate the unwanted pixels from the original image. The whole steps of the preprocessing are showed in algorithm 1 below:

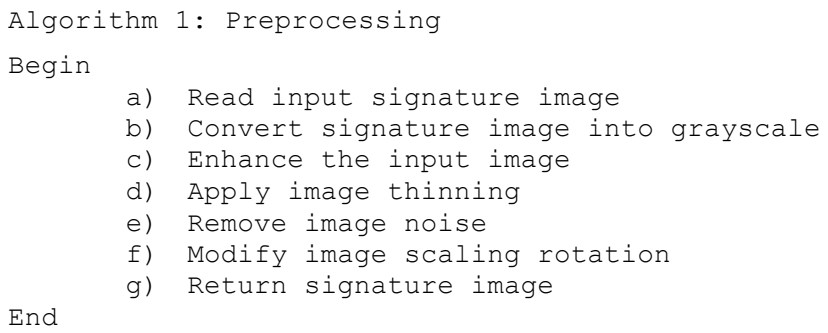

Thinned image still represent the signature shape within few pixels. Thus, make the computation process is fast and accurate in the same time. However, 128x128 image size is selected in our work after many experiments for better verification results. The effects of applying the preprocessing on the input signature image are shown in Figure 2.

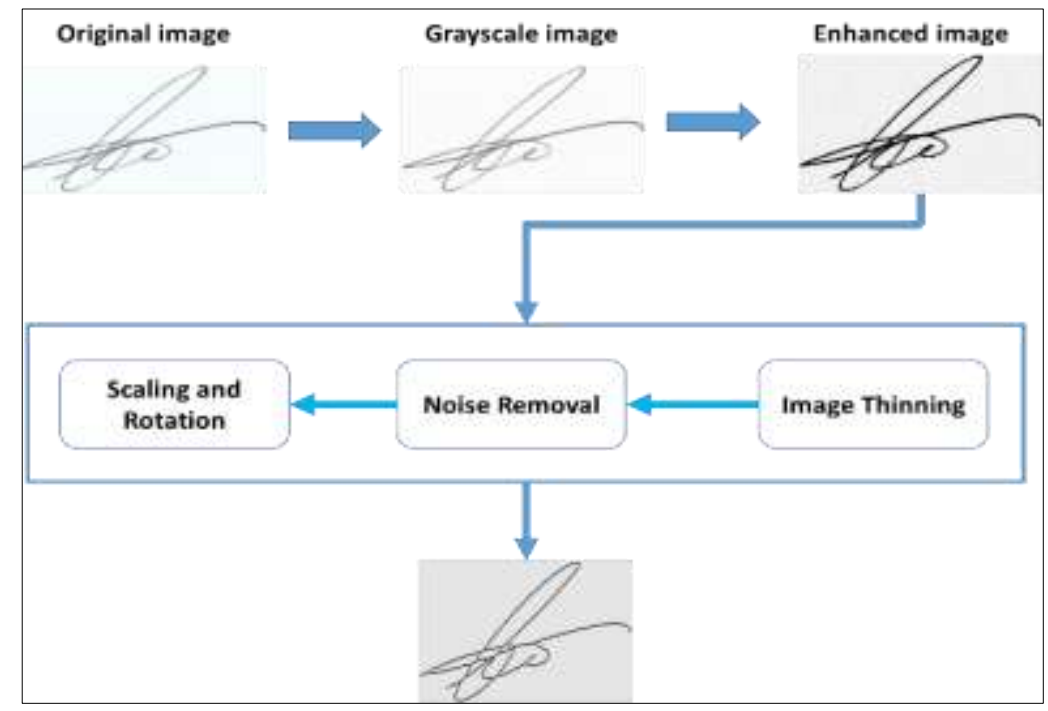

Figure 2. Preprocessing of proposed work

\subsection{Feature extraction}

The most effective stage of the verification system is the feature extraction. Using an appropriate feature extraction methods leads the system for efficient and accurate verification results. In the proposed work, a new approach for extracting the desired features is proposed. The approached used the histogram of sparse codes (HSC) [18] for extracting the signature features in the efficient manner. The full steps of feature extraction process are shown in algorithm 2. A visualization of the feature extraction process in the proposed wok is shown in Figure 3.

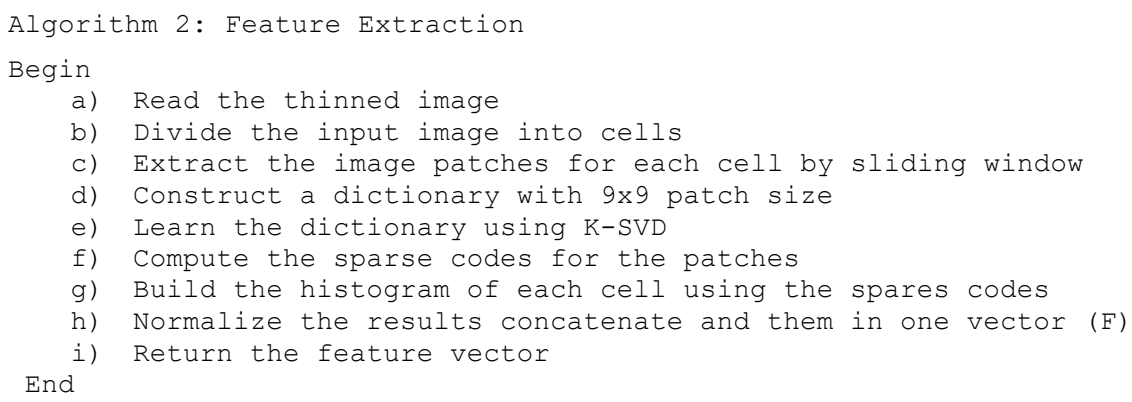




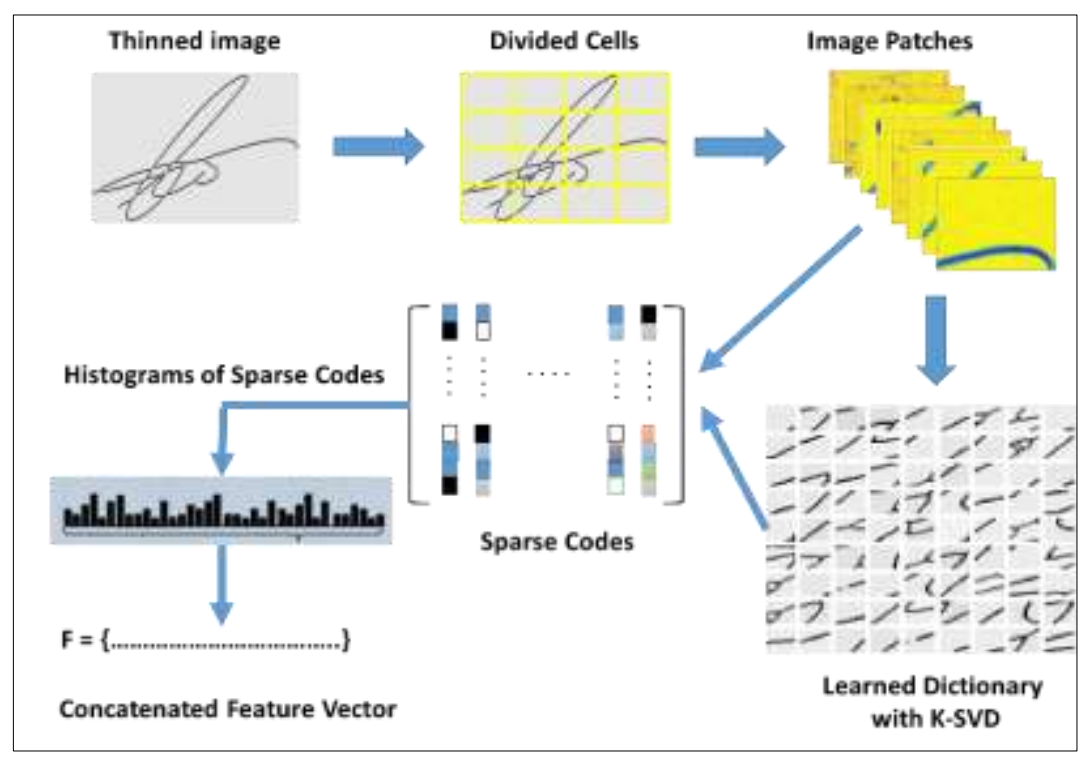

Figure 3. Proposed feature extraction

HSC is inspired by histogram of oriented gradient (HOG) descriptor. It applied in the object detection systems an achieved a better detection results than HOG [19]. It represent each image patch with a set of atoms the sparse dictionary. Then sparse codes are aggregated into histograms on regular cells to generate feature vectors [20]. Let the set of image patches $\mathrm{Y}=[\mathrm{y} 1, \ldots, \mathrm{yn}], \mathrm{K}-\mathrm{SVD}$ [17] is used for learning by jointly fin the dictionary $\mathrm{D}=[\mathrm{d} 1, \ldots, \mathrm{dm}]$, the matrix of sparse codes $\mathrm{X}=[\mathrm{x} 1, \ldots, \mathrm{xn}]$, via minimize the following reconstruction error:

$$
\min _{D, X}\|Y-D X\|_{2}^{f} \text { s.t. } \forall_{i},\left\|x_{i}\right\|_{0} \leq K
$$

Where

$\mathrm{X}_{\mathrm{i}}$ columns of $\mathrm{X}$

$\|.\|_{0}$ non zero entries

$\mathrm{K}$ the sparsity level

This optimization can be solved by alternating between computing X and D using K-SVD. Also, orthogonal matching pursuit (OMP) [21] is efficiently used for computing the codes X. The dictionary $D$ is updated sequentially for the given the codes $\mathrm{X}$, using singular value decomposition. Furthermore, OMP is used again for computing the sparse code at every image pixels after the dictionary $\mathrm{D}$ is learned. Thus, a considerable speed-up is provided by the precomputing the inner products between patches and codewords as in [22]. The soft binning is used for $x_{i}$ in $X$. The results a features vector $F$ which is normalized with its L2 norm. Then, in order to make the values more uniform, a power transform is applied for F elements as in (2):

$$
\bar{F}=F^{\propto}
$$

Although HSC provide an accurate results, it produce a long feature vector [vertical cells $\mathrm{x}$ horizontal cells $\mathrm{x}$ dictionary elements]. In our proposed work a thinned image with small amount of dictionary elements are used and preformed fast and accurate in the verification. Moreover, the constructed vector will include only the best angles which are [30, 45, 60, 90, and 180] that match the most effective sparse codes of the input signatures and ignore the other sparse.

\subsection{Classification}

In the last stage of our verification system is to classify the signature as a genuine or forgery. Using proper classifier among the available classifiers can be increase the system performance. Therefore, in the proposed work, artificial neural networks (ANN) [23] is used for classification and make the final decision. In our system the ANN have three layers which are input, hidden and output layers. Multilayer feed forward network (with Backpropagation algorithm)is used for indicating whether the signature is fake or true using 
100 inputs, two 40 hidden neurons, and 2 outputs. In the training phase 24 signature for each person are used, 12 true signatures and 12 fake signatures. The best variables for training the networks was: 0.4 for learning rate, with 1 as an initial weight. However, in the testing face, 100 signature images are used, 50 as a true signatures and 50 as a fake signatures. The proposed ANN architecture illustrated in Figure 4.

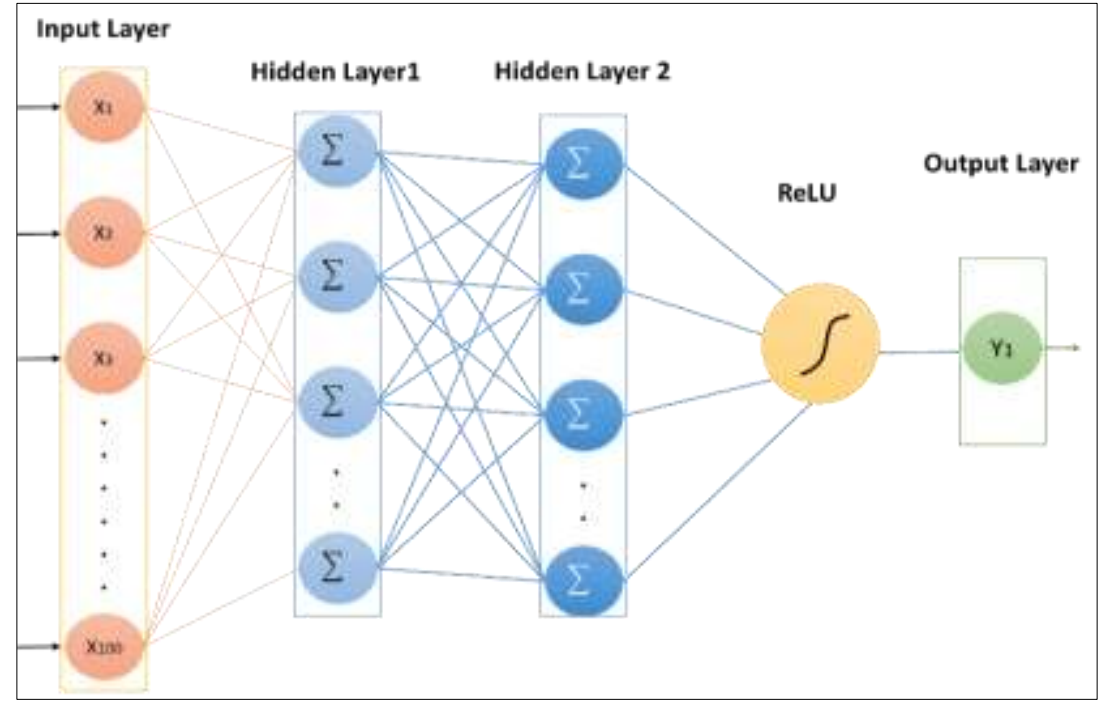

Figure 4. Proposed ANN architecture

\section{RESULTS AND DISCUSSION}

Matlab 2019b under windows 10 (64-bit) environment is used for implementation the proposed system. Intel core i5 processor, 8 GB memory, with machine learning library of Matlab employed in our system. Besides, three signatures datasets are used for evaluation the proposed system which are CEDAR [24], UTSig [25], and ICDAR [26-27]. A sample signature images of the used datasets are shown in Figure 5.

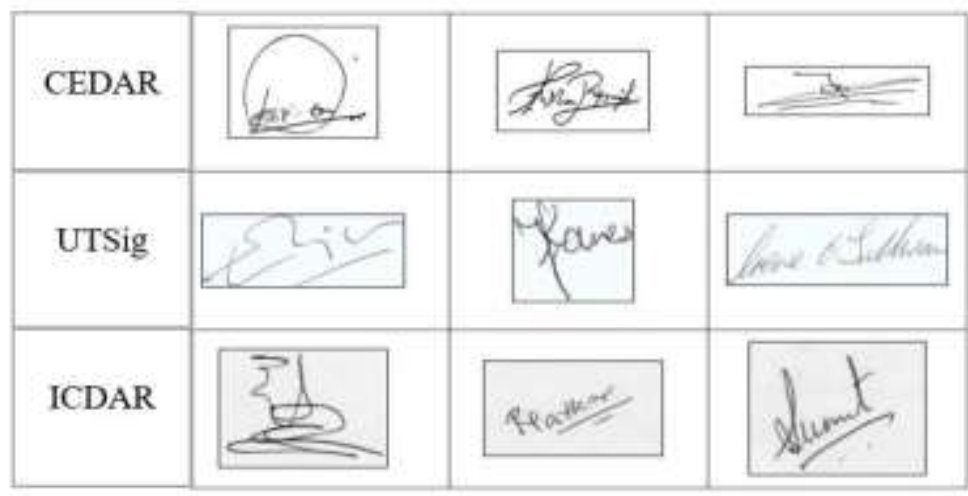

Figure 5. Samples of the used signature images

In the proposed feature extraction approach based on HSC, we use the edged image and the thinned image to extract the features and evaluating the results. The extracted patches, dictionary and sparse codes from the thinned image is faster and accurate than the edge image which is observed via many experiments. An example of the built dictionaries using the two type of images are shown if Figure 6.

Chossing the appropertate activation function is convergence of the neural net that largely depends on. ReLU is yhe most effective activation function used in hidden layers of neural network. It works by thresholding values at 0 , i.e. $(x)=\max (0, x)$. It simply outputs 0 when $x<0$ while outputs a linear function when $x \geq 0$. 


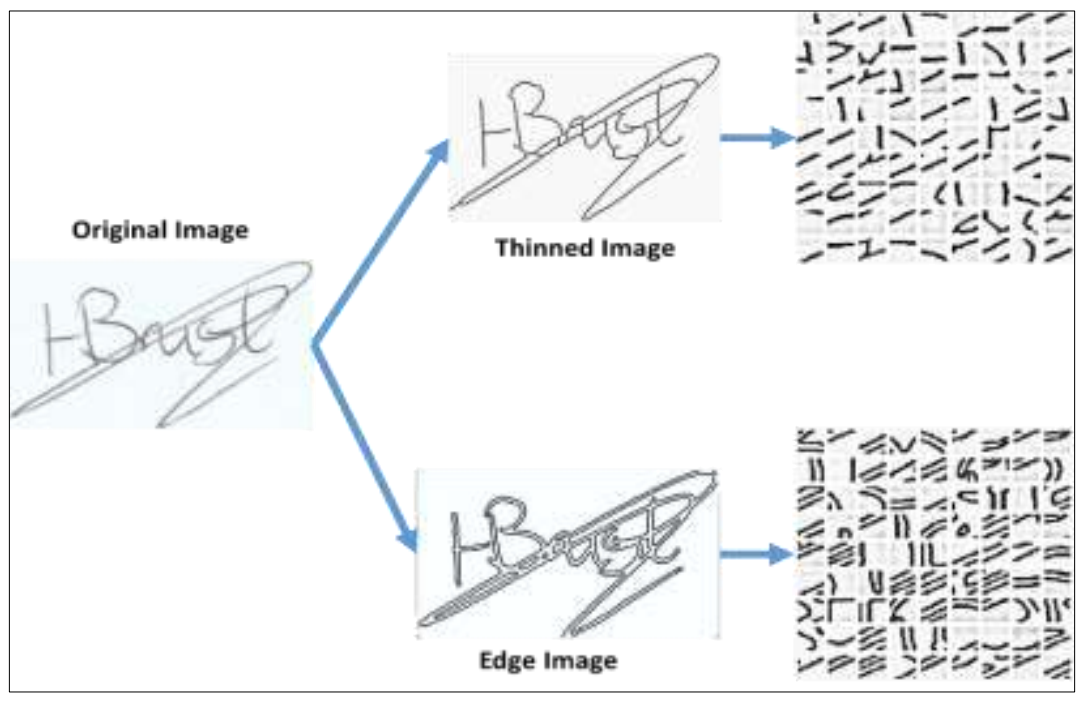

Figure 6. Learned dictionaries for thinned and edge images

The verification results and computation time of the proposed system for the both images are viewed in Table 1. In addition, different dictionary sizes are tested in order to get the most appropriate size. In the proposed system $9 \times 9$ in chosen after performing many experiments. The grown in dictionary size is given more features for the signature like line endings, corners, and high-frequency gratings, thin lines. The archived results for different dictionary sizes are shown in Table 2 and an example of the dictionary images are in Figure 7.

Table 1. Verification results of different image types

\begin{tabular}{ccc}
\hline Image Type & Accuracy $(\%)$ & Time (ms.) \\
\hline Thinned Images & 99.7 & 0.09 \\
Edge Images & 97.2 & 0.29 \\
\hline
\end{tabular}

Table 2. System results for various dictionary sizes

\begin{tabular}{cc}
\hline Dictionary Size & Accuracy $(\%)$ \\
\hline $3 \times 3$ & 95.6 \\
$5 \times 5$ & 96.1 \\
$8 \times 8$ & 98 \\
$9 \times 9$ & 99.7 \\
\hline
\end{tabular}

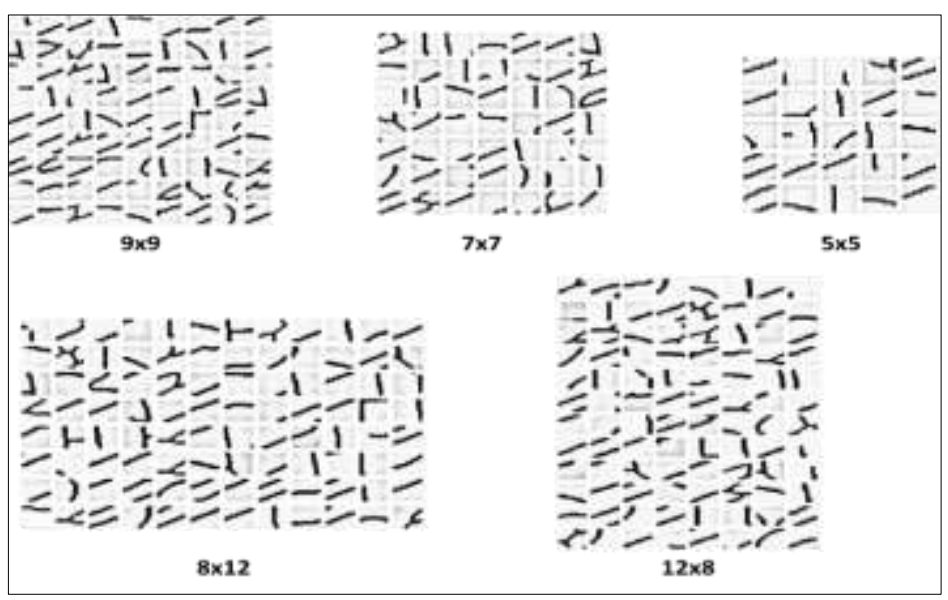

Figure 7. Learned dictionaries with different sizes

HCS did not employed in previous verification systems. Most of the recent and previous systems are used HSC in object recognition. HSC improved the accuracy in many recognition system. Therefore, in your system HSC is applied sufficiency for best possible results. Besides, the proposed approach of using the HSC leaded the system to obtain a high verification accuracy than obtained by the standard HSC as in Table 3 . 
In another hand, proposed classification architecture give a high verification results comparing to SVM and KNN classifier which are tested in our system. In Figure 8 the obtained results of the three mentioned classifiers are shown.

Table 3. Comparison results of HSC method and proposed method

\begin{tabular}{cc}
\hline Method & Accuracy $(\%)$ \\
\hline Standard HSC & 96.1 \\
Proposed & 99.7 \\
\hline
\end{tabular}

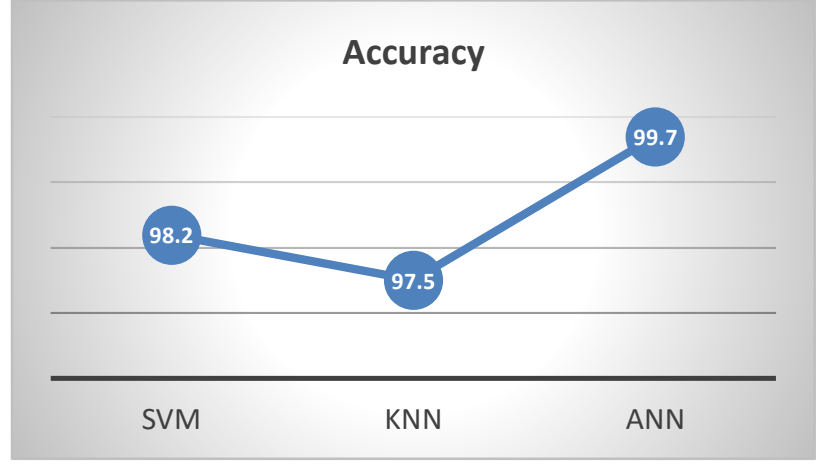

Figure 8. Verification results by ANN, SVM and KNN

The proposed system performance is showed reliable results via high verification accuracy and fast computing speed. A comparison of our system results and the state of arts results are shown in Table 4. In addition, rectified linear unit (ReLU) activation function is employed sufficiently in the proposed syste. ReLU has achived the best results in various systems with ANN and CNNs. In our system, the importance of using ReLU leads the system for fast and accurate results comparing to other activation functions. The results of applying ReLU activation function in the proposed system is shown in Table 5.

Table 4. Comparison results of proposed system and other systems

\begin{tabular}{cc}
\hline Method & Accuracy (\%) \\
\hline sCNN [11] & 93 \\
CNN [12] & $90-94$ \\
SNN [14] & 88.49 \\
SVM [15] & 89.58 \\
ANN [27] & 89.24 \\
Proposed & 99.7 \\
\hline
\end{tabular}

Table 5. Comparison results of various activation functions

\begin{tabular}{ccc}
\hline Function & Time $(\mathrm{ms})$. & Accuracy $(\%)$ \\
\hline Sigmoid & 0.51 & 99 \\
Hyperbolic & 0.24 & 98.8 \\
Tangent & 0.09 & 99.7 \\
ReLU & \\
\hline
\end{tabular}

\section{CONCLUSION}

Fast, accurate and reliable signature verification system is proposed in this paper. The system achieved a very high verification accuracy which is $99.7 \%$ and it's higher than the existing systems. Besides, the used preprocessing techniques within the proposed feature extraction approach based on HSC leaded the system to get a better accuracy results. Furthermore, the proposed ANN architecture enhance the obtained results with minimum errors based on three public different signatures image databases. In the future work, CNNs could be employed effecinty in real time verification system for feature extraction and classification using wighted Softmax activation function which is succsussfully appied in many identification and recognition systems.

\section{REFERENCES}

[1] L. G. Hafemann, R. Sabourin, and L. S. Oliveira, "Learning features for offline handwritten signature verification using deep convolutional neural networks," Pattern Recognition, vol. 70, pp. 163-176, 2017.

[2] M. B. Yilmaz and K. O" ztu"rk, "Hybrid user-independent and userdependent offline signature verification with a two-channel cnn," 2018 IEEE/CVF Conference on Computer Vision and Pattern Recognition Workshops (CVPRW), pp. 639-6398, 2018.

[3] Manoj Chavan, Ravish R. Singh, Vinayak Bharadi, "Online signature verification using hybrid wavelet transform", International Journal of Electrical and Computer Engineering (IJECE), vol. 10, no. 2, pp. 1823-183, 2020.

[4] Javier O. Pinzón-Arenas, Robinson Jiménez-Moreno, César G. Pachón-Suescún, "Offline signature verification using DAG-CNN", International Journal of Electrical and Computer Engineering (IJECE), vol. 9, no. 4, pp. 33143322, 2019. 
[5] Duc Nguyen Tan, Hai Nguyen Nam, Minh Nguyen Hieu, "Blind multi-signature scheme based on factoring and discrete logarithm problem”, TELKOMNIKA, vol. 17, no. 5, pp. 2327-2334, 2019.

[6] L. G. Hafemann, R. Sabourin, and L. S. Oliveira, "Offline handwritten signature verification-Literature review," in Seventh International Conference on Image Processing Theory, Tools and Applications (IPTA), 2017, pp. 1-8, 2017.

[7] Kadhm, M.S. and Hassan, A.K.A., "Handwriting word recognition based on SVM classifier," International Journal of Advanced Computer Science \& Applications, vol. 1, pp. 64-68, 2015.

[8] Shaymaa A., Abdulrahman Wael Khalifa, Mohamed Roushdy, Mohamed Roushdy, Abdel-Badeeh, "Comparative study for 8 computational intelligence algorithms for human identification," Computer Science Review, Elsevier, vol. 36, pp. 1-9, 2020.

[9] M. B. Y1lmaz and B. Yanıko glu, "Score level fusion of classifiers in off-line signature verification," Information Fusion, vol. 32, Part B, pp. 109-119, 2016.

[10] L. G. Hafemann, R. Sabourin, and L. S. Oliveira, "Learning features for offline handwritten signature verification using deep convolutional neural networks," Pattern Recognition, vol. 70, pp. 163-176, 2017.

[11] Jain, A., Singh, S.K. \& Singh, K.P. "Handwritten signature verification using shallow convolutional neural network," Multimed Tools Applm, 2020.

[12] Jivesh P., Vinanti P., and Santosh K., "Offline Signature Recognition and Forgery Detection using Deep Learning," The 3rd International Conference on Emerging Data and Industry 4.0 (EDI40), Warsaw, Poland, Procedia Computer Science, Elsevier, vol. 170, pp. 610-617, 2020.

[13] Tomasz H., and Jerzy P., "Long-term verification of signatures based on a blockchain," Computers and Electrical Engineering, Computers \& Electrical Engineering, Elsevier, vol. 81, 2020.

[14] Jagtap, A.B., Sawat, D.D., Hegadi, R.S. et al. "Verification of genuine and forged offline signatures using Siamese Neural Network (SNN)," Multimed Tools Appl, 2020.

[15] Pradeep N., Rajendra R., and Sanjiv V., "Offline signature verification using shape correspondence," Int. J. Biometrics, Inderscience, vol. 10, no. 3, pp. 272-289, 2018.

[16] Victoria R., Ismael L., Angel S., and JoseF. Velez, "Off-line handwritten signature verification using compositional synthetic generation of signatures and Siamese Neural Networks," Neurocomputing, Elsevier, vol. 374, pp. 30-41, 2019.

[17] Hassan, A.K.A. and Kadhm, M.S., "An Efficient Preprocessing Framework for Arabic Handwriting Recognition System," Diyala Journal For Pure Science, vol. 12, no. 3-part 2, pp. 147-163, 2016.

[18] X. Ren, and D. Ramanan, "Histograms of sparse codes for object detection," in: IEEE Computer Vision and Pattern Recognition, pp. 3246-3253, 2013.

[19] Dong Zhang, Da-Han Wang, and Hanzi Wang, "Scene Text Recognition Using Sparse Coding Based Features," IEEE International Conference on Image Processing (ICIP), pp. 1066-1070, 2014.

[20] M. Aharon, M. Elad, and A. Bruckstein, "K-SVD: An Algorithm for Designing Overcomplete Dictionaries for Sparse Representation,” IEEE Transactions on Signal Processing, vol. 54, no. 11, pp. 4311-4322, 2006.

[21] Y. Pati, R. Rezaiifar, and P. Krishnaprasad., "Orthogonal Matching Pursuit: Recursive Function Approximation with Applications to Wavelet Decomposition," In The Twenty-Seventh Asilomar Conference on Signals, Systems and Computers, pp. 40-44, 1993.

[22] R. Rubinstein, M. Zibulevsky, and M. Elad., "Efficient Implementation of the K-SVD Algorithm using Batch Orthogonal Matching Pursuit," Technical report, CS Technion, 2008.

[23] Jayanta Kumar Basu, Debnath Bhattacharyya, Taihoon Kim, "Use of Artificial Neural Network in Pattern Recognition", International Journal of Software Engineering and Its Applications, vol. 4, no. 2, pp. 24-32, 2010.

[24] Souza, V. L. F., Oliveira, A. L. I., Cruz, R. M. O., \& Sabourin, "R. (2019b). 845 On dissimilarity representation and transfer learning for offline handwritten signature verification," In 2019 International Joint Conference on Neural Networks (IJCNN), 2019.

[25] Amir Soleimani, Kazim Fouladi, and Babak N. Araabi, "UTSig: A Persian offline signature dataset," IET Biometrics, vol. 6, no. 1, pp.1-8, 2017.

[26] Marcus Liwicki, Michael Blumenstein, Elisa van den Heuvel, Charles E.H. Berger, Reinoud D. Stoel, Bryan Found, Xiaohong Chen, Muhammad Imran Malik., "SigComp11: Signature Verification Competition for On- and Offline Skilled Forgeries," Proc. 11th Int. Conference on Document Analysis and Recognition, 2011.

[27] Subhash Chandra, and Sushila Maheskar, "Offline signature verification based on geometric feature extraction using artificial neural network," 3rd Int'l Conf. on Recent Advances in Information Technology, 2016.

\section{BIOGRAPHIES OF AUTHORS}

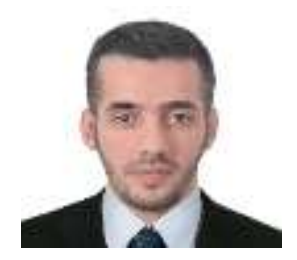

Mustafa Salam is an associate dean at the faculity of information technology, Imam Ja'afar AlSadiq University. He received his B.S. degrees in Software Engineering from Al-Mansour University College, Baghdad, Iraq in 2009 and M.S. in Information Technology from University of Tun Abdulrazak, Malaysia in 2012. In 2017 he recive the $\mathrm{PhD}$. in computer science from university of technology, Iraq. His research interests include Artificial Intelligence, Image Processing, Computer Vision, Pattern Recognition, and Data Mining. Email: muit.salam@sadiq.edu.iq. 


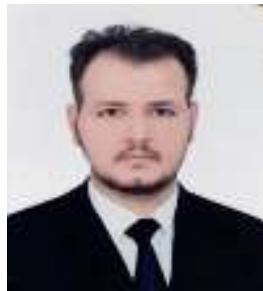

Mamoun Jassim Mohammed is a lecturer at Department of Computer Engineering, College of Engineering, Al-Iraqia University, in Baghdad, Iraq. He recive his master degree in computer cience from university of Baghdad. Email: mamoun_87@yahoo.com, mamoun.jassim@aliraqia.edu.iq.

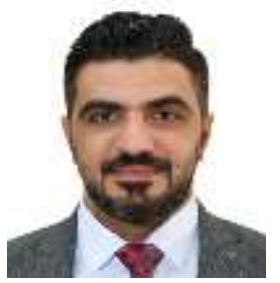

Hayder Ayad currently works at the College of Business Administration, Al-Bayan University. Their current project is 'Visual object categorization'. He recived his $\mathrm{PhD}$. in computer science from university of technology, Iraq, 2017. Email: Hayder.alsultany@ gmail.com. 